\title{
Médiévales
}

Langues, Textes, Histoire

59 | automne 2010

Théâtres du Moyen Âge

\section{Arthur chez les historiens}

\section{Michelle Szkilnik}

\section{(2) OpenEdition}

Journals

Édition électronique

URL : https://journals.openedition.org/medievales/6192

DOI : 10.4000/medievales.6192

ISSN : $1777-5892$

\section{Éditeur}

Presses universitaires de Vincennes

\section{Édition imprimée}

Date de publication : 31 décembre 2010

Pagination : 171-181

ISBN : 978-2-84292-267-2

ISSN : 0751-2708

\section{Référence électronique}

Michelle Szkilnik, "Arthur chez les historiens », Médiévales [En ligne], 59 | automne 2010, mis en ligne le 12 mars 2011, consulté le 24 avril 2022. URL : http://journals.openedition.org/medievales/6192 : DOI : https://doi.org/10.4000/medievales.6192

Ce document a été généré automatiquement le 24 avril 2022

Tous droits réservés 


\title{
Arthur chez les historiens
}

\author{
Michelle Szkilnik
}

\section{RÉFÉRENCE}

Alban Gautier, Arthur, Paris, 2007

Martin Aurell, La légende du roi Arthur, Paris, 2007

Amaury Chauou, Le Roi Arthur, Paris, 2009

1 L'intérêt pour le personnage d'Arthur et ses chevaliers ne s'est jamais démenti depuis le Moyen Âge, mais il semble étonnamment vif en ce début du $\mathrm{xxI}^{\mathrm{e}}$ siècle. La production de films récents ${ }^{1}$ et le succès en France de la série télévisée Kamelott témoignent de l'attrait que la légende exerce sur un large public, attrait qu'alimente et dont tire profit la communauté des chercheurs, historiens et littéraires. Deux expositions ont été récemment consacrées à Arthur : l'une s'est tenue à Rennes du 15 juillet 2008 au 4 janvier 2009, en marge du Congrès triennal de la Société Internationale de Littérature Arthurienne, la seconde à la Bibliothèque Nationale de France, site Tolbiac, du 20 octobre 2009 au 24 janvier 2010. Une troisième, en lien avec les deux autres, est en cours de préparation à la médiathèque de Troyes. Les expositions de Rennes et Paris avaient pour objet de retracer la naissance, le développement au Moyen Âge et l'évolution jusqu'à nos jours de la légende, à travers la littérature, l'art, voire le cinéma pour la période moderne. Celle de la Bibliothèque Nationale a donné lieu à la publication d'un magnifique catalogue intitulé La Légende du roi Arthur ${ }^{2}$, dont la direction a été assurée par Thierry Delcourt, commissaire de l'exposition, conservateur $\mathrm{du}$ cabinet des manuscrits et bon connaisseur de la littérature arthurienne ${ }^{3}$. Ont contribué au catalogue des médiévistes d'horizons divers : spécialistes des manuscrits, spécialistes de littérature, historiens et historiens de l'art. Abondamment illustré, l'ouvrage contient des articles bien informés, destinés à un large public, ainsi que des notices de manuscrits, de sorte qu'il offre de l'intérêt à la fois pour des lecteurs peu familiers avec la légende arthurienne dans la mesure où il retrace de manière claire l'histoire des principaux personnages et motifs, et pour les chercheurs du fait de l'abondance des illustrations et des descriptions de manuscrits. Son format, une 
succession d'articles brefs rédigés par un spécialiste sur un sujet précis - fabrication et illustration des manuscrits arthuriens, Chrétien de Troyes, le Graal, l'héraldique arthurienne pour n'en citer que quelques-uns -, met tour à tour en lumière une facette de la légende dont la totalité se construit ainsi par vignettes juxtaposées. Le catalogue est complété par un site Internet encore accessible ${ }^{4}$ qui propose en particulier une section "livres à feuilleter " permettant de consulter neuf manuscrits partiellement numérisés.

2 Tout autres sont la forme et la visée de trois livres récents consacrés au roi breton: $L a$ Légende du roi Arthur de Martin Aurell, Arthur d'Alban Gautier et Le Roi Arthur d'Amaury Chauou ${ }^{5}$. Écrits par des historiens, ces ouvrages se proposent d'embrasser l'ensemble de la matière arthurienne depuis le $\mathrm{VI}^{\mathrm{e}}$ siècle jusqu'à nos jours pour les deux derniers, de 550 à 1250 pour le livre de M. Aurell. C'est dire qu'à côté de documents historiques et archéologiques, ils exploitent des sources essentiellement littéraires. À la différence d'un livre comme celui de R. Morrissey sur Charlemagne qui se veut une «mythistoire " de l'empereur à la barbe fleurie dans le domaine français, c'est-à-dire une histoire qui retrace les différentes appropriations de la figure contribuant à la définition de l'identité française ${ }^{6}$, l'ambition des trois livres sur Arthur me paraît plus large et l'histoire des appropriations politiques de la légende n'est qu'un aspect d'une somme qui entend aussi rendre compte de la puissance du mythe dans l'imaginaire collectif.

Pour la spécialiste de littérature que je suis et qui aurait tendance à considérer qu'Arthur appartient plutôt à mon domaine, ces livres posent ultimement la question du public auquel ils sont destinés. Ce sont, me semble-t-il, des ouvrages de vulgarisation au meilleur sens du terme. Ils visent un public large, certes éduqué mais n'ayant que des connaissances vagues ou en tout cas partielles de la matière arthurienne. Sur bien des points ils reprennent et résument les recherches sur Arthur menées tout au long du $\mathrm{xx}^{\mathrm{e}}$ siècle, en grande partie du reste par des spécialistes de littérature. Corollairement on peut s'interroger sur la part de liberté que leurs auteurs ont eue dans leur conception. S'il parait clair que M. Aurell a mené son entreprise à sa guise, qu'il a disposé d'un nombre de pages très confortable et a volontairement choisi de croiser l'histoire et la littérature sur une période relativement restreinte, A. Gautier et A. Chauou n'ont-ils pas dû se plier à un format qui les a obligés à sortir et de leur champ méthodologique et de leur champ temporel?

\section{Écrire une biographie d'Arthur?}

4 Alors que l'existence historique de Charlemagne est avérée, ce qui laisse à l'historien le choix d'écrire une biographie ou une histoire des représentations de l'empereur au fil du temps, Arthur pose un tout autre problème: comment parler en historien d'un personnage dont on ne sait pas s'il a vraiment existé et dont la mémoire est conservée dans des sources majoritairement littéraires? S'il était prouvé et sans doute possible qu'Arthur est un personnage historique, un historien pourrait écrire sa biographie même à partir de sources limitées et peu fiables. Si Arthur n'était qu'un personnage de fiction, comme Tristan ou Lancelot, les historiens l'abandonneraient sans état d'âme aux littéraires. Aucun historien n'a jamais tenté d'écrire la biographie du roi Marc, l'oncle de Tristan. Mais qui est Arthur? Ou, question plus pressante, Arthur a-t-il existé? Alors que les spécialistes de littérature peuvent éluder l'interrogation pour se concentrer sur la biographie fictive du roi, les historiens qui écrivent sur Arthur 
doivent nécessairement la soulever. Et ils doivent y répondre en s'appuyant sur des sources à la fois trop et trop peu abondantes: trop dans la mesure où les mentions d'Arthur dans les textes écrits, mais aussi dans l'art, sont extrêmement nombreuses tout au long du Moyen Âge; trop peu parce que les témoignages anciens sont quasi inexistants : quelques traces archéologiques, quelques allusions dans des textes dont on peut supposer qu'ils conservent le souvenir des $\mathrm{v}^{\mathrm{e}}$ et $\mathrm{vi}^{\mathrm{e}}$ siècles.

5 Face à cette difficulté, ou bien on se refuse à l'exercice biographique après avoir montré qu'il est impossible, ou bien on tient le pari à l'aide des rares documents existants. M. Aurell et A. Chauou ont choisi la première solution, A. Gautier la seconde.

6 Les titres respectifs de leurs ouvrages et le choix de l'illustration de couverture en orientent déjà la lecture. Le Roi Arthur, d'A. Chauou, dont la couverture représente un jeune cavalier, aux traits androgynes, magnifiquement vêtu, se baissant élégamment pour empoigner l'épée fichée dans un curieux perron, tout en tenant d'une main ferme son fougueux coursier, invite d'emblée à voir dans Arthur un personnage de légende. L'image est une illustration de Cheri Herouard pour Les Chevaliers de la Table Ronde de Ronan Caerleon, réalisée en 1945. Le titre de roi conféré à Arthur, l'image de l'épée qui lui est associée sont des éléments légendaires qu'aucune source historique ne vient confirmer. Le titre et l'illustration sont donc des indices d'une lecture mythique du destin d'Arthur. Le parti pris est encore plus clair pour le livre de M. Aurell, La Légende du roi Arthur: l'introduction du mot légende dans le titre souligne immédiatement le caractère fabuleux de la tradition qui entoure le personnage d'Arthur. La couverture reproduit une enluminure tirée de la Chronique anglo-normande en vers de Pierre de Langtoft (début xiv $V^{e}$ siècle), représentant un roi en majesté, assis sur un trône, couronné et tenant dans chacune de ses mains une couronne, tandis que deux autres couronnes se détachent encore sur le fond. L'enluminure insiste donc sur la royauté du personnage. Choisir une enluminure tirée d'une chronique engage à penser que le livre va privilégier la dimension politique d'Arthur. Quant à l'ouvrage d'A. Gautier, simplement intitulé Arthur, il utilise également en couverture une enluminure de la Chronique anglo-normande de Pierre de Langtoft. Elle figure Arthur en roi guerrier portant un bouclier à l'effigie de la Vierge Marie, image qui rappelle l'un des épisodes les plus anciens liés au personnage: la victoire qu'Arthur aurait remportée au Mont Badon, grâce au soutien de la Vierge. Or, cette bataille est l'un des rares événements un peu assurés dans cette période mal connue. Ce choix engage implicitement le lecteur dans la quête d'une dimension historique d'Arthur. Par ailleurs, l'enluminure qui montre un roi couronné entre en tension avec le titre qui ne donne que le nom du personnage, ce qui problématise d'entrée de jeu la question de la royauté d'Arthur.

7 Comment les trois historiens confrontent-ils l'absence de sources historiques fiables garantissant l'existence d'Arthur?

8 Dans le chapitre intitulé «L'énigme d'Arthur", A. Chauou pose en termes clairs la question de l'historicité d'Arthur: Arthur est-il un personnage historique autour duquel s'est édifiée une légende ou bien un personnage légendaire qui s'est vu attribuer au fil du temps une réalité historique ${ }^{7}$ ? De manière très significative, ce chapitre ne figure pas au début du livre qui s'ouvre sur un parcours à travers les hauts lieux de la légende arthurienne et continue par un rapide tableau du personnel arthurien. C'est dire que pour A. Chauou l'historicité d'Arthur n'est finalement pas un sujet essentiel. Il faut bien l'aborder, mais pour l'écarter rapidement. Récapitulant les témoignages de l'archéologie ainsi que ceux des textes anciens qui font allusion à Arthur ou à un chef 
de guerre en lutte contre les Anglo-Saxons, A. Chauou passe en revue les différentes théories qui soutiennent l'existence d'un Arthur historique (celle de Geoffrey Ashe, celles élaborées à la suite des travaux de Rachel Bromwich) pour conclure qu'aucune n'est satisfaisante et qu'on ne peut prouver l'historicité d'Arthur ${ }^{8}$.

9 Conformément à la promesse de son titre, $M$. Aurell s'intéresse lui aussi très peu à cette question. Il semble même d'abord l'ignorer purement et simplement. L'introduction à sa première partie s'ouvre en effet sur ces mots: "Arthur n'a vraisemblablement jamais existé ", puis évoque « la vie fictive » du personnage et la " genèse fabuleuse » de ce "mythe " ${ }^{9}$. Ce sont en effet la naissance et le développement d'une légende qu'il souhaite retracer et les différents usages qui en ont été faits. Quelles sont les formes de la légende et qui en a profité, voilà les questions qu'il entend plutôt traiter. Il est vrai qu'il revient sur le problème dans un bref développement intitulé «Arthur a-t-il existé? Une affaire d'anthroponymie ${ }^{10}$. Après avoir étudié les membres de l'élite guerrière ayant porté le nom d'Arthur au début du Moyen Âge, M. Aurell conclut qu'en attribuant le nom Arthur à ses enfants, l'aristocratie des $\mathrm{VI}^{\mathrm{e}}$ et $\mathrm{VII}^{\mathrm{e}}$ siècles cherchait à s'approprier le prestige d'un mythe «local». Un peu plus loin, il rejette clairement toute existence historique d'Arthur, préférant voir dans le roi un personnage de fiction, «l'avatar tardif d'un héros mythologique, faisant désormais partie des récits folkloriques et figé dans la topographie ${ }^{11}$.

Seul A. Gautier relève la gageure : écrire la biographie d'Arthur sans preuve aucune de son existence historique. Cette tentative qui pourrait paraître absurde est en réalité plutôt réussie. À défaut de pouvoir donner du roi une biographie assurée, A. Gautier brosse d'Arthur un portrait plausible en s'appuyant sur des informations rares, certes, mais vérifiables, concernant des personnages historiques, contemporains du supposé chef militaire. Le chapitre VI, "Possible biographie d'un possible Arthur", est une fascinante "tentative de reconstitution de la carrière d'un chef de guerre dans la Bretagne des Âges Obscurs " ${ }^{12}$. À partir du postulat indémontrable: Arthur a bien existé (presque un acte de foi demandé au lecteur, une suspension of disbelief, comme disent les Anglo-Saxons), A. Gautier nous explique ce qu'il a dû être. À cette occasion, c'est un tableau vif et passionnant des $\mathrm{V}^{\mathrm{e}}$ et $\mathrm{vI}^{\mathrm{e}}$ siècles qu'il peint, tableau dans lequel se dessine en creux la silhouette d'Arthur : grand chef de guerre au tournant du vi siècle, Arthur aurait, aux côtés de rois bretons, repoussé à plusieurs reprises les Saxons. Ayant mené les troupes chrétiennes à la victoire au siège de Badon, il s'entoura d'une fidèle bande de guerriers, réunissant autour de lui une cour plus proche de celle d'un " aristocrate romain du début du $v^{\text {e }}$ siècle " que de celle d'un roi médiéval ${ }^{13}$. S'ensuivit une longue période de paix avec les Saxons, mais de guerres fratricides entre Bretons. C'est durant l'une d'elles qu'Arthur affronte un certain Medraut et qu'il succombe lors d'un combat. Comme A. Chauou, A. Gautier croise témoignages archéologiques et sources textuelles, mais tout en reconnaissant ce que ces documents ont de douteux et de contradictoire, il utilise les quelques certitudes qu'ils contiennent pour élaborer sa construction qu'il présente toujours prudemment comme un modèle possible. C'est là la grande différence avec les théories, surtout anglo-saxonnes, critiquées par A. Chauou, et qui visent à prouver l'existence historique d'Arthur. A. Gautier sait qu'il ne prouve rien mais il dit à son lecteur: si Arthur a existé, voilà à quoi il ressemblait. Rédigée par un spécialiste de ces Âges Obscurs, cette biographie possible se pare de tous les attraits non de la vérité mais de la vraisemblance historique. 


\section{Du bon usage des sources littéraires}

11 Les sources concernant Arthur sont peu abondantes pour les périodes anciennes. Pour les $\mathrm{VI}^{\mathrm{e}}$ - $\mathrm{XI}^{\mathrm{e}}$ siècles, outre des découvertes archéologiques peu faciles à interpréter, nous disposons de quelques témoignages textuels eux-mêmes problématiques. Des textes à caractère historique écrits en latin: la Décadence de la Bretagne de Gildas, rédigée au début du $\mathrm{VI}^{\mathrm{e}}$ siècle, L'Histoire de Bretons de Nennius, compilation de textes datant des $\mathrm{IX}^{\mathrm{e}}$ $\mathrm{X}^{\mathrm{e}}$ siècles, parmi lesquels les Annales de Cambrie qui couvrent les années 453-954; un ensemble de cinq vies de saints, également rédigées en latin, écrites dans les années 1100-1130; enfin des poèmes ou brefs récits en langue galloise transmis par oral jusqu'au XIII ${ }^{e}$ siècle, où ils sont mis par écrit. On comprend d'emblée la difficulté d'utiliser des sources qui d'abord, du fait même de leur nature, font la part belle à la fiction, ensuite nous sont conservées dans des manuscrits copiés plusieurs siècles après la date supposée de leur élaboration. Les vies de saints par exemple racontent les exploits de pieux personnages ayant vécu aux $\mathrm{v}^{\mathrm{e}}-\mathrm{vl}^{\mathrm{e}}$ siècles, comme Gildas ou Padarn, mais elles sont mises en forme au début $\mathrm{du} \mathrm{xI}^{\mathrm{e}}$ siècle. Sans doute reprennent-elles des données transmises oralement, mais la forme, la rhétorique, voire le type d'anecdotes des œuvres sont tributaires de modèles hagiographiques plus tardifs et extrêmement prégnants, de sorte qu'il est difficile d'apprécier, derrière l'épais écran de la construction littéraire, ce qui peut renvoyer à une réalité ancienne. Quant aux textes poétiques, si les contraintes formelles qui les caractérisent (les triades en langue cymrique en particulier) peuvent être une garantie de leur ancienneté, il n'en demeure pas moins que leur caractère fragmentaire et leur visée lyrique font obstacle à une exploitation historique. Même les sources en apparence plus historiques sont d'utilisation difficile à cause de leur nature hybride et les événements qu'elles consignent n'ont pas nécessairement une réalité historique avérée. Ces difficultés sont cependant le lot de tout historien travaillant sur les périodes anciennes et aux franges de sociétés plus brillantes. N'étant pas spécialiste des littératures celtiques anciennes, je ne peux pas juger de la manière dont les trois historiens ont utilisé les sources à leur disposition et je préfère donc me concentrer sur ce que je connais bien mieux : les XII et $\mathrm{XIII}^{\mathrm{e}}$ siècles.

12 Exploiter les textes littéraires médiévaux, les historiens l'ont fait et doivent le faire. Jacques Le Goff, pour ne citer que lui, a montré de manière magistrale comment les romans de Chrétien pouvaient être mis à profit ${ }^{14}$. Cette exploitation suppose une connaissance approfondie et personnelle des textes sans laquelle l'historien est condamné à reprendre des lieux communs ou des hypothèses dépassées, voire à perpétuer les erreurs qui traînent dans des manuels de littérature. Peut-on encore affirmer par exemple que Cligès est «le seul roman [de Chrétien de Troyes] qui n'appartient pas à la matière de Bretagne ${ }^{15}$ alors que de très nombreux épisodes du roman se déroulent à la cour d'Arthur?

13 L'un des écueils les plus difficiles à éviter peut-être est celui d'une histoire littéraire dont les spécialistes de littérature ont vu depuis longtemps les limites. S'efforcer de fournir des informations sur la vie d'auteurs dont l'existence est parfois discutée, sans prendre en compte les recherches actuelles qui s'interrogent précisément sur la pertinence des notions d'auteur et d'œuvre pour la période médiévale, n'est-ce pas céder à la tentation d'un modèle dépassé ? La Légende du roi Arthur contient un chapitre intitulé «Vie et œuvre de Chrétien de Troyes», dans la plus pure tradition des manuels 
d'histoire littéraire. Après avoir fait le point sur les rares éléments biographiques que les chercheurs ont pu réunir sur Chrétien de Troyes, avoir revisité la chronologie de ses œuvres, il s'intéresse tour à tour à chacun des romans de Chrétien dont il donne d'abord un long résumé avant de proposer quelques pistes d'interprétation. Ce chapitre est indiscutablement une bonne synthèse des travaux récents ou plus anciens, réalisés par les critiques littéraires sur l'œuvre de Chrétien. Destiné à un public qui connaît mal Chrétien de Troyes, il ne procède toutefois pas d'une approche proprement historique qui renouvellerait notre connaissance de ces textes ${ }^{16}$.

14 A. Gautier et A. Chauou consacrent quant à eux un long développement au personnel de la légende arthurienne (respectivement «Les hommes (et les femmes) d'Arthur » et "À la table ronde d'Arthur »). Sans doute est-il utile de guider à travers cette forêt de noms un public peu familier avec la matière de Bretagne. Tous deux dessinent donc des cercles concentriques autour d'Arthur sur lesquels ils placent les différents héros dont ils brossent rapidement la biographie. Le danger de cette méthode est bien sûr de simplifier grandement la réalité textuelle. L'exemple le plus net est le cas d'Arthur luimême. Lorsqu'A. Chauou, par exemple, prétend « résum [er] la célèbre matrice laissée par Geoffroy de Monmouth et, dans une certaine mesure, par Wace ${ }^{17}$, il prête à Geoffroy des épisodes qui ne figurent pas dans l'Historia Regum Britanniae. Dans ce texte, et chez Wace également, si Arthur naît bel et bien d'une liaison illégitime entre Uterpendragon et la duchesse de Tintagel, il n'est toutefois jamais soupçonné de bâtardise puisque le duc de Tintagel étant mort fort opportunément la nuit de la conception d'Arthur, le roi épouse sur le champ la duchesse, de sorte qu'au moment où l'enfant naît, personne n'imagine qu'il a pu être conçu hors des liens du mariage. À la mort d'Uter, les chefs bretons se réunissent à Silchester où ils demandent à l'archevêque Dubricius de sacrer roi Arthur, ce qui est fait sans contestation aucune ni signe divin quelconque en faveur d'Arthur. Le célébrissime épisode de l'épée dans le perron n'existe donc ni chez Geoffroy, ni chez Wace, où il n'a aucune raison d'être. Certes, A. Chauou le signale dans une note, mais il a auparavant raconté que l'enfant Arthur est enlevé par Merlin à ses parents et confié à Antor qui l'élève dans l'ignorance de son identité, de sorte qu'à la mort d'Uter s'ouvre une crise de succession. Or, c'est tout cet ensemble qui ne figure pas chez Geoffroy, non plus bien sûr que les guerres civiles qui déchirent le royaume de Logres après l'élection d'Arthur. Ce n'est qu'à partir du Merlin en prose du pseudo Robert de Boron, et dans les suites de ce texte que l'on rencontre ces développements qui se sont pourtant imposés comme la vérité sur Arthur.

Le personnage de Tristan offre un autre exemple du danger d'élaborer la biographie fictive d'un héros en se fondant sur des récits divers, écrits à des époques différentes. Tristan, qu'A. Gautier et A. Chauou placent légitimement aux marges du monde arthurien, est loin d'être une figure unifiée et dire à son propos que «ce sont les aventures des amants éternels [...] qui intéressent avant tout l'auditoire [...], bien plus que la qualité de chevalier de la Table Ronde $»^{18}$ n'est absolument pas juste en ce qui concerne le Tristan en prose. De même, il est faux d'affirmer que « Tristan et Yseult sont l'image même de l'amour courtois, à la fois absolu et adultère ${ }^{19}$. Victimes de la passion amoureuse, ils sont loin d'être dans les premiers textes de fins amants ${ }^{20}$.

De texte en texte, d'époque à époque, de pays en pays, les héros arthuriens se modifient considérablement. Tracer à grands traits la biographie fictive d'Arthur ou d'autres personnages arthuriens, c'est courir le risque d'ignorer la spécificité des textes et la 
plasticité des personnages de fiction qui, tout déterminés qu'ils soient par la tradition, n'en subissent pas moins parfois des altérations étonnantes. Qu'on songe par exemple à l'image que Valentin et Orson, roman de la fin du Moyen Âge, donne d'Arthur : il fait du roi légendaire un traître qui veut détrôner Pépin, épouser Berthe et exiler le petit Charlot, futur Charlemagne. Emprisonné dans la prison du Châtelet, Arthur meurt misérablement, décapité par Pépin ${ }^{21}$.

La légende arthurienne s'incarne dans des textes de formes diverses qui répondent à des exigences variées. Ce n'est pas un ensemble fixé au terme d'une évolution linéaire et simple, mais un conglomérat mouvant, réinterprété et modifié à chaque reprise de la légende. Chaque texte re-conte Arthur, c'est-à-dire conte son histoire à sa manière.

\section{Pour une lecture politique des textes littéraires}

18 Là où les trois livres font en revanche une lecture spécifiquement historique et très éclairante des sources littéraires, c'est quand ils examinent la dimension politique de la légende. Il faut donner un sens large à ce mot politique qui recouvre également la portée religieuse et idéologique que les auteurs médiévaux confèrent à la légende.

Parce qu'il s'intéresse à un laps de temps plus circonscrit (quoique très long), M. Aurell peut, pour chaque période considérée, examiner en détail à quel usage les différents auteurs ont asservi Arthur. L'exercice de la royauté, les valeurs et comportements du groupe chevaleresque que reflètent par exemple les romans de Chrétien (M. Aurell s'engage ici dans la voie ouverte par Jean Flori ${ }^{22}$ ), l'idéal religieux dont sont imprégnés les romans du Graal en prose et la volonté de conversion qui les anime, autant de terrains où l'historien peut croiser le spécialiste de littérature et développer une " histoire sociale, politique et mentale de la fiction ${ }^{23}$ ". L'analyse que M. Aurell donne du manteau d'Érec lors de son couronnement par exemple complète et corrige de manière originale et convaincante la lecture traditionnelle de cette description bien connue des littéraires ${ }^{24}$.

20 L'un des points sur lesquels les historiens ont depuis longtemps exploité avec succès le roman arthurien est celui des rapports entre la cour d'Arthur et celle des Plantagenêts. Les rois d'Angleterre ont en effet essayé de capter le prestige de la légende. Pour A. Chauou qui synthétise dans deux chapitres ("Arthur et les Anglo-Normands", "L'idéologie arthurienne à la cour Plantagenêt») les recherches qu'il avait consacrées à la royauté arthurienne et à la monarchie politique ${ }^{25}$, c'est surtout à partir de Richard Cœur de Lion que le roi d'Angleterre essaie de se présenter comme un digne descendant d'Arthur: "l'invention» de la tombe d'Arthur à Glastonbury en 1191, le choix d'une bannière portant un dragon qui renvoie au roi légendaire Uterpendragon, le don d'Excalibur, l'épée d'Arthur, au roi de Sicile Tancrède sont autant de témoignages du rattachement au mythe arthurien. En ce qui concerne l'épée, on peut comme Jean Flori s'interroger sur le don à un allié de second rang d'un tel « symbole de la dignité royale ${ }^{26}$ » et du coup relativiser le poids de la légende. J. Flori suggère finement que des considérations matérielles sont peut-être venues contrebalancer les préoccupations de prestige et d'image. Représentant la prouesse et le pouvoir, l'épée n'en est pas moins l'objet que s'approprient le plus volontiers les princes et rois désireux de célébrer leur lien avec d'illustres chevaliers arthuriens. A. Chauou rappelle ainsi que Jean Sans Terre, qui avait combattu en Irlande et avait reçu en apanage l'Irlande et la Cornouaille, tente de s'octroyer la gloire de Tristan: il avait, semble-t-il, 
fait forger pour son couronnement une épée au tranchant volontairement entaillé d'une encoche imitant celle que Tristan endommagea lors de son combat contre le Morholt.

21 M. Aurell examine lui aussi l'utilisation que les rois d'Angleterre ont faite de la figure d'Arthur et des prophéties de Merlin détournées ou plus exactement refaçonnées de manière à justifier la conquête de l'Irlande et de l'Écosse ${ }^{27}$. Alors que sous Henri II, Arthur reste encore largement le roi breton porteur des espoirs celtiques et donc potentiellement hostile à la nouvelle dynastie angevine, à partir du XIII ${ }^{e}$ siècle, Arthur perd son «localisme" pour devenir roi d'Angleterre et servir les intérêts des Plantagenêts. A. Gautier montre de même comment Arthur devient pour des raisons politiques un héros pan-brittonique, avant de procurer aux Plantagenêts un modèle et bientôt un ancêtre ${ }^{28}$.

Si les hommes politiques du Moyen Âge ont tenté de s'accaparer la force de la légende transmise en particulier par les textes littéraires, les écrivains de leur côté se sont fait l'écho des préoccupations politiques de leur temps, prêtant ainsi au roi Arthur et à sa cour des comportements et des aspirations qui sont ceux de la société aristocratique qu'ils connaissaient. L'exemple le plus commenté et le plus frappant est sans doute celui de la fête de couronnement d'Érec à Noël dans la ville de Nantes : telle qu'elle est décrite par Chrétien de Troyes, elle évoque irrésistiblement les festivités intervenues dans la capitale bretonne à la Noël 1169, au cours desquelles les barons bretons prêtèrent hommage à Geoffroy Plantagenêt fiancé à Constance, héritière du comté de Bretagne ${ }^{29}$. Les analogies repérées par les historiens permettent d'imaginer que Chrétien de Troyes s'est rendu en Angleterre à la cour d'Henri II et fournissent par ailleurs un élément de datation pour Érec et Énide.

Ces dernières années les relations entre historiens et spécialistes de la littérature se sont multipliées et on ne peut que s'en féliciter. Cette collaboration est fructueuse si chacun conserve la spécificité de son approche et fait parler les textes avec les méthodes de son champ disciplinaire, comme le rappelle justement $\mathrm{M}$. Aurell qui, dans son introduction, souligne la supériorité de la "pluridisciplinarité " («échanges intenses entre branches différentes du savoir») sur l'interdisciplinarité (qui vise "à constituer un champ de savoir unique ${ }^{30}$ ). Il me semble cependant qu'Arthur pose une redoutable difficulté à qui veut pratiquer cette collaboration souhaitable, car il existe un fort déséquilibre entre les branches du savoir concernées: à mon avis, seule une approche littéraire (elle-même complexe et multiple) peut pleinement rendre compte des constructions diverses dont le personnage est le centre. L'approche historique est condamnée à demeurer incomplète parce qu'elle ne peut pas expliquer la force du mythe par la somme des utilisations ponctuelles qui en ont été faites. Arthur a certes incarné les espoirs du peuple breton et servi le prestige de la dynastie Plantagenêt; certes les romans qui racontent l'histoire de ses chevaliers reflètent des réalités sociales et politiques, et certes, il est précieux de connaître tout cela. Mais la raison d'être d'Arthur et de sa cour, le rôle qu'il joue aujourd'hui encore dans l'imaginaire excèdent immensément les captations dont il a été l'objet. Je dirais que ce sont des épiphénomènes qu'il est important d'apprécier mais qui ne suffisent pas pour comprendre « l'invention » d'Arthur. 


\section{NOTES}

1. Dernier en date, King Arthur d'Antoine Fuqua, réalisé en 2004, qui se prétend la "véritable » histoire du roi Arthur.

2. La Légende du roi Arthur, sous la direction de Thierry Delcourt, Paris, Bibliothèque nationale de France/Seuil, 2009.

3. Il a rédigé le Que Sais-Je? sur la littérature arthurienne (T. DELCOURT, La Littérature arthurienne, Paris, 2000).

4. T. DElCourt, S. TOUlouse, Arthur,1. De l'histoire à la littérature, Bibliothèque nationale de France, 2009, http://expositions.bnf.fr/arthur/expo/salle1/index.htm [consulté le 3 octobre 2010].

5. A. GAUTIER, Arthur, Paris, 2007. M. AURell, La Légende du roi Arthur, Paris, 2007. A. ChaUou, Le Roi Arthur, Paris, 2009.

6. Voir R. MORRISSEY, L'Empereur à la barbe fleurie. Charlemagne dans la mythologie et l'histoire de France, Paris, 1997.

7. A. CHAUOU, p. 118.

8. Ibid., p. 120-121.

9. M. AURELL, op. cit., p. 35.

10. Ibid.,p. 93-96.

11. Ibid.p. 98.

12. A. GAUTIER, op. cit, p. 145 .

13. Ibid., p. 169.

14. On citera par exemple «Lévi-Strauss en Brocéliande. Esquisse pour une analyse d'un roman courtois » qui porte sur le Chevalier au lion et «Codes vestimentaire et alimentaire dans Érec et Énide », tous deux republiés dans L'Imaginaire médiéval, Paris, 1985 (respectivement p. 151-187 et 188-207).

15. A. GAUTIER, op. cit., p. 236.

16. Voir aussi A. Gautier sur Chrétien de Troyes, op. cit., p. 235-238.

17. A. CHAUOU, op. cit., p. 63.

18. A. CHAUOU, op. cit., p. 92.

19. A. GAUTIER, op. cit., p. 308.

20. Voir les bonnes mises au point d'E. Baumgartner dans Tristan et Iseut, Paris, 1987 et Tristan et Iseut, Paris, 2001.

21. Voir A. DICKSON, Valentine et Orson, a Study in Late Medieval Romance, New York, 1929, p. 237-239.

22. Parmi les très nombreux travaux que Jean Flori a consacrés à ce sujet, on citera simplement «La notion de chevalerie dans les romans de Chrétien de Troyes », Romania, 114, 1996, p. 289-315, ainsi que Chevaliers et chevalerie au Moyen Âge, Paris, 1998.

23. M. AURELL, op. cit., p. 33.

24. Ibid., p. 306-309.

25. A. CHAUOU, L'Idéologie Plantagenêt. Royauté arthurienne et monarchie politique dans l'espace Plantagenêt (XII ${ }^{e}$-XIII ${ }^{e}$ siècles), Rennes, 2001.

26. J. FLORI, Richard Cour de Lion. Le Roi-chevalier, Paris, 1999, p. 473.

27. Voir son quatrième chapitre : « Henri II, ses fils et la légende arthurienne », p. 165-209.

28. Voir chapitre $X$, «La Matière de Bretagne », en particulier p. 228-234.

29. M. AURELL, op. cit., p. 182-185, A. CHAUOU, op. cit., p. 163-164, A. GAUTIER, op. cit., p. 237-238.

30. M. AURELL, op. cit., p. 30-31. 
INDEX

Mots-clés : Arthur, biographie, historiographie, littérature

Keywords : Arthur, biography, historiography, literature

\section{AUTEURS}

\section{MICHELLE SZKILNIK}

Université de Paris 3-Sorbonne Nouvelle, 17 rue de la Sorbonne, 75231 Paris cedex 05 